\title{
The complex of hypericin with $\beta$-lactoglobulin has antimicrobial activity with potential applications in dairy industry
}

\author{
Beatriz Rodríguez-Amigo, ${ }^{*}$ Pietro Delcanale, $†$ Gabriel Rotger, ${ }^{*}$ Jordi Juárez-Jiménez,ł \\ Stefania Abbruzzetti,§\# Andrea Summer,Il Montserrat Agut, ${ }^{*}$ F. Javier Luque,‡ \\ Santi Nonell, ${ }^{*}$ and Cristiano Viappiani†\# ${ }^{1}$ \\ *Institut Quimic de Sarrià, Universitat Ramon Llull, Via Augusta 390, 08017 Barcelona, Spain \\ †Dipartimento di Fisica e Scienze della Terra, Università di Parma, Viale delle Scienze 7A, 43124 Parma, Italy \\ fDepartament de Fisicoquímica and Institut de Biomedicina, Facultat de Farmàcia, Universitat de Barcelona, Avda. Prat de la Riba 171, \\ 08921 Santa Coloma de Gramenet, Spain \\ §Dipartimento di Bioscienze, Università di Parma, Viale delle Scienze 11A, 43124 Parma, Italy \\ \#NEST, Istituto Nanoscienze, CNR, Piazza San Silvestro 12, 56127 Pisa, Italy \\ IIDipartimento di Scienze degli Alimenti, Università di Parma, via del Taglio, 10, 43126 Parma, Italy
}

\begin{abstract}
Using a combination of molecular modeling and spectroscopic experiments, the naturally occurring, pharmacologically active hypericin compound is shown to form a stable complex with the dimeric form of $\beta$-lactoglobulin ( $\beta$-LG). Binding is predicted to occur at the narrowest cleft found at the interface between monomers in the dimeric $\beta$-LG. The complex is able to preserve the fluorescence and singlet oxygen photosensitizing properties of the dye. The equilibrium constant for hypericin binding has been determined as $K_{\mathrm{a}}=1.40$ $\pm 0.07 \mu M^{-1}$, equivalent to a dissociation constant, $K_{\mathrm{d}}=0.71 \pm 0.03 \mu M$. The complex is active against Staphylococcus aureus bacteria. Overall, the results are encouraging for pursuing the potential application of the complex between hypericin and $\beta-\mathrm{LG}$ as a nanodevice with bactericidal properties for disinfection.
\end{abstract}

Key words: hypericin, $\beta$-lactoglobulin, photosensitizer, antimicrobial

\section{INTRODUCTION}

Antimicrobial products are important for the health and welfare of livestock, but prudent use is necessary. In fact, the overuse of antibiotics can generate drugresistant bacteria, with the possibility of transferring them to the food chain (IDF, 2013). For example, methicillin-resistant Staphylococcus aureus (MRSA) is a well-known food-borne pathogen that produces heat-stable enterotoxins during growth on a variety of foods and is an important regional cause of human infection (Doyle et al., 2011). Recently, it has been noted

Received August 1, 2014.

Accepted October 4, 2014.

${ }^{1}$ Corresponding author: cristiano.viappiani@fis.unipr.it that livestock animals can be reservoirs of MRSA and transmit it to humans that live in close contact with them; MRSA from animals is called livestock-associated MRSA (Graveland et al., 2011). For this reason, it is important to develop new alternative strategies for preventing and treating infectious animal diseases of bacterial origin (Trevisi et al., 2014). Indeed, development of novel methods for decontamination of food processing and handling environment is a key topic for food science (Demirci and Ngadi, 2012; Kairyte et al., 2012; Luksiene and Brovko, 2013).

Antibacterial photosensitization-based treatment (APBT) is an emerging methodology that relies on the combined action of an otherwise nontoxic molecule (called photosensitizer), visible light, and oxygen to produce cytotoxic effects by the photoinduced generation of reactive oxygen species (photodynamic effect), particularly singlet oxygen $\left({ }^{1} \mathrm{O}_{2}\right)$. Thus, photoexcitation of the photosensitizers leads to the formation of ${ }^{1} \mathrm{O}_{2}$, a nonradical, electronically excited form of the dioxygen molecule that is highly reactive against a vast array of cellular components ranging from membrane lipids to proteins and nucleic acids (Ogilby, 2010; Jori et al., 2011). The APBT has proved to be valuable for the treatment of localized microbial infections, effectively acting on several classes of microbial pathogens without inducing insurgence of photoresistant species even after multiple treatments. The selectivity of microbial cell killing in comparison with the constituent host tissues, and the possibility to activate photodynamic sensitizers by means of inexpensive and safe visible light sources, are further elements in favor of the methodology (Jori et al., 2011).

Hypericin (Hyp; Figure 1) is a natural product, structurally belonging to the chemical class of naphthodianthrones, which is found in plants from the genus Hypericum (e.g., St. John's wort; Brockmann et al., 
1939; Durán and Song, 1986). St. John's wort infusions are being used as herbal aids for depression and Hyp has also been used as an antiviral (Jacobson et al., 2001; Kubin et al., 2005), antibacterial (Kairyte et al., 2012; Yow et al., 2012), and antifungal agent (Rezusta et al., 2012). The antimicrobial photoactivity of Hyp against MRSA, both in vitro and in vivo, has been recently assessed (Nafee et al., 2013). Whereas Hyp is largely insoluble in water, it dissolves readily in ethanol or dimethylsulfoxide, where it displays a bright red color due to its absorption and fluorescence emission properties (Durán and Song, 1986; López-Chicón et al., 2012). In these solvents, Hyp is also a strong photosensitizer, with singlet oxygen quantum yields exceeding 0.7 (Michaeli et al., 1993; Fehr et al., 1995). When dissolved in water, Hyp tends to form aggregates, which results in broadening of the bands in the absorption spectrum in a much weaker, broad, and structureless fluorescence emission, and loss of singlet oxygen photosensitizing efficiency (Yamazaki et al., 1993).

Because the effectiveness of Hyp-mediated photodynamic action is heavily dependent on the specific localizing capability of the delivery methodology, proteins appear as particularly interesting delivery agents thanks to their high biocompatibility, bioavailability, and the possibility to select specific proteins suitable to the environment to be targeted. We have recently shown that Hyp binds to the hydrophobic pocket of apomyoglobin, preserving its fluorescence and ${ }^{1} \mathrm{O}_{2}$ photosensitizing properties (Comas-Barceló et al., 2013). Furthermore, we have shown that the complex is active against Staphylococcus aureus bacteria.

In the current study, we propose the use of $\beta-\mathrm{LG}$, because it is the most abundant protein in the whey of cow milk (Flower et al., 2000), as a delivery vehicle for antimicrobial photodynamic application of Hyp. $\beta$-Lactoglobulin is a small, homodimeric protein of 162 amino acids $(\sim 18,400 \mathrm{Da})$ belonging to the lipocalin

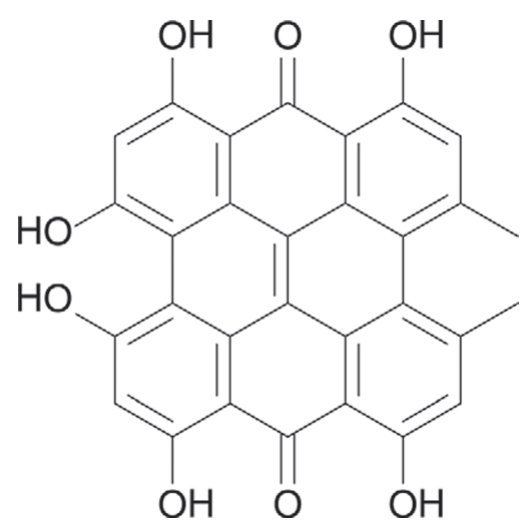

Figure 1. Chemical structure of hypericin. family (Åkerström et al., 2006; Mercadante et al., 2012). Owing to their ability to bind small hydrophobic molecules, while simultaneously being recognized by cell surface receptors, lipocalins were suggested as potential systems for drug delivery (Åkerström et al., 2006). Whereas some lipocalins possess high ligand specificity, others, such as $\beta$-LG, demonstrate a remarkable versatility in their ligand-binding patterns. Affinity for small molecules is generally intermediate, with dissociation constants $\left(K_{d}\right)$ around $1 \mu M$. We used the B variant of $\beta$-LG, characterized by glycine and alanine at positions 64 and 118 (Tanford and Nozaki, 1959), to examine the ability to bind Hyp and the effect of binding on the photodynamic properties of Hyp. Additionally, we show that the complex between $\beta$-LG and Hyp is able to inactivate Staph. aureus bacteria. In view of the biocompatibility of the $\beta$-LG carrier with dairy products as well as with milk processing and handling material, this construct has potential for photodynamic antibacterial applications in the dairy industry.

\section{MATERIALS AND METHODS}

Hypericin was obtained from HWI Analytik GmbH (Ruelzheim, Germany). $\beta$-Lactoglobulin (isoform B) from bovine milk was obtained from Sigma Aldrich (St. Louis, MO). All samples were used as received.

\section{Docking Calculations}

The protein model for the bovine $\beta$-LG dimer was derived from the X-ray crystallographic structure (PDB entry 1BEB, http://www.rcsb.org/; Brownlow et al., 1997). The structure was refined by including addition of hydrogen atoms using the xLEAP facility in AMBER12 (Case et al., 2012) and the parm99SB (Hornak et al., 2006) force field, removal of nonstandard residues (sulfate anion), and generation of the disulfide bonds Cys66-Cys160 and Cys106-Cys119. Because the N- and C-terminal segments are not observed in the X-ray structure, N-methylamine and acetyl capping groups were added to the $\mathrm{C}$ - and $\mathrm{N}$-terminus, respectively. The binding of Hyp was explored by means of docking calculations carried out with GLIDE (Friesner et al., 2004; Halgren et al., 2004). Because the size of Hyp precludes its binding to the cavity shaped in the interior of the lipocalin-type $\beta$-barrel, docking was performed in the 2 pockets formed at the interface of the monomers in the dimeric complex. The first pocket included the solventexposed residues inside a cube of $39,300 \AA^{3}$, which was centered between side chains of residues Arg148 of one monomer and Asp137 of the other. The second cavity was defined by the exposed residues inside a cube of the same size centered at the midpoint of the segment 
that connects the side chains of residue Trp61 in each monomer.

\section{General Spectroscopic Instrumentation}

Absorption spectra recorded using a Varian Cary 6000i (Varian Inc., Palo Alto, CA). Fluorescence spectra were recorded using a Spex-Fluoromax 4 (Horiba Jobin Yvon, Edison, NJ) or a Perkin Elmer LS50 spectrofluorometers (PerkinElmer, Waltham, MA).

\section{Singlet Oxygen Detection}

Production of the reactive species singlet oxygen was assessed by monitoring its specific phosphorescence at $1,275 \mathrm{~nm}$ with a setup described in detail elsewhere (Jiménez-Banzo et al., 2008). Hypericin was excited by a pulsed laser at $532 \mathrm{~nm}$ and the emission of singlet oxygen was detected by a dedicated near infrared photomultiplier.

\section{Microbial Strains and Growth Conditions}

Staphylococcus aureus CECT 239 was obtained from the Spanish Type Culture Collection (CECT). Bacterial cells were grown overnight in sterile tryptic soy broth at $37^{\circ} \mathrm{C}$. Stock inoculum suspensions were prepared in PBS and adjusted to optical densities of 0.4 at $600 \mathrm{~nm}$.

\section{Photodynamic Inactivation of Staph. aureus}

Cell suspensions in PBS were incubated with the photosensitizer for $30 \mathrm{~min}$ in the dark at room temperature. Then, $0.3 \mathrm{~mL}$ of the suspensions were placed in 96 -well plates. The plates were illuminated from the top by use of light-emitting diode green light for 15 or $30 \mathrm{~min}$ (18 and $37 \mathrm{~J} / \mathrm{cm}^{2}$, respectively), then serially diluted, seeded on tryptic soy agar and incubated for $24 \mathrm{~h}$ at $37^{\circ} \mathrm{C}$. Experiments were carried out in duplicate for each condition, including cell controls and dark controls. Colony-forming units were counted to calculate the survival fraction.

\section{RESULTS AND DISCUSSION}

\section{Modeling of $\beta-L G-H y p$ interaction}

$\beta$-Lactoglobulin forms dimers (2 $\beta$-LG) under conditions close to those encountered physiologically, with a dimerization constant of $K_{D}=8.6 \mu \mathrm{M}$ at $\mathrm{pH} 7.5$ (Mercadante et al., 2012). $\beta$-Lactoglobulin is shaped to accommodate small hydrophobic compounds, such as retinol and the aliphatic chains of FA, in the lipocalintype $\beta$-barrel. However, the cavity is too narrow to ac- commodate Hyp, which suggests that this compound should bind other pockets. In particular, inspection of the dimeric structure of $\beta-L G$ revealed that Hyp might bind to the clefts formed at the interface between monomers (Figure 2). The analysis of the best-ranked poses supported the binding of Hyp to the narrow cleft, as the predicted score $(-7.4 \mathrm{kcal} / \mathrm{mol})$ is $\sim 2.5 \mathrm{kcal} / \mathrm{mol}$ more favorable than the binding to the wide cleft (score of $-4.9 \mathrm{kcal} / \mathrm{mol}$ ). This finding is not unexpected, given the hydrophobicity of the compound and the narrow separation between the walls of this cleft, where Нyp is largely occluded from the solvent. In contrast, binding to the wide cleft leaves one of the 2 faces of Hyp exposed to the solvent (Figure 2).

\section{Spectroscopic Analysis of Hyp Binding to $\beta-L G$}

Binding between Hyp and $2 \beta-\mathrm{LG}$ can be monitored through the effects on the absorption spectrum and fluorescence emission. Aqueous solutions of Hyp show broad absorption bands and very weak and structureless fluorescence emission (Figure 3A; Miskovsky et al., 1998). When Hyp is dissolved in the presence of $2 \beta-L G$, its absorption spectrum shows sharper bands instead and, most importantly, the fluorescence emission increases substantially and becomes structured (Figure 3B). These facts indicate that Hyp is located in a substantially less polar environment than water, as previously observed with apomyoglobin and human serum albumin (Miskovsky et al., 1998; Das et al., 1999; Comas-Barceló et al., 2013), as a result of binding to the protein. These conditions are a prerequisite for preservation of the singlet oxygen photosensitization via energy transfer from the Hyp triplet state. A more detailed characterization of the photophysical properties of the complex is beyond the scope of the present work and will be reported elsewhere.

As virtually only bound Hyp molecules are fluorescent, the recorded fluorescence emission intensity is proportional to the fraction of bound molecules. Accordingly, the affinity of Hyp for $2 \beta-L G$ can be evaluated by monitoring the fluorescence emission as a function of Hyp concentration (Figure 3C). A model for the binding equilibrium Hyp $+2 \beta-\mathrm{LG} \leftrightarrows 2 \beta-\mathrm{LG}-$ Hyp (Comas-Barceló et al., 2013) affords an association constant, $K_{a}=1.40 \pm 0.07 \mu M^{-1}$, corresponding to a dissociation constant, $K_{d}=0.71 \pm 0.03 \mu M$.

\section{Singlet Oxygen Production}

Production of the reactive species singlet oxygen was demonstrated by observation of its specific phosphorescence at 1,275 $\mathrm{nm}$. Figure 4 shows the intensity of the singlet oxygen emission of Hyp and of the Hyp- $2 \beta-\mathrm{LG}$ 


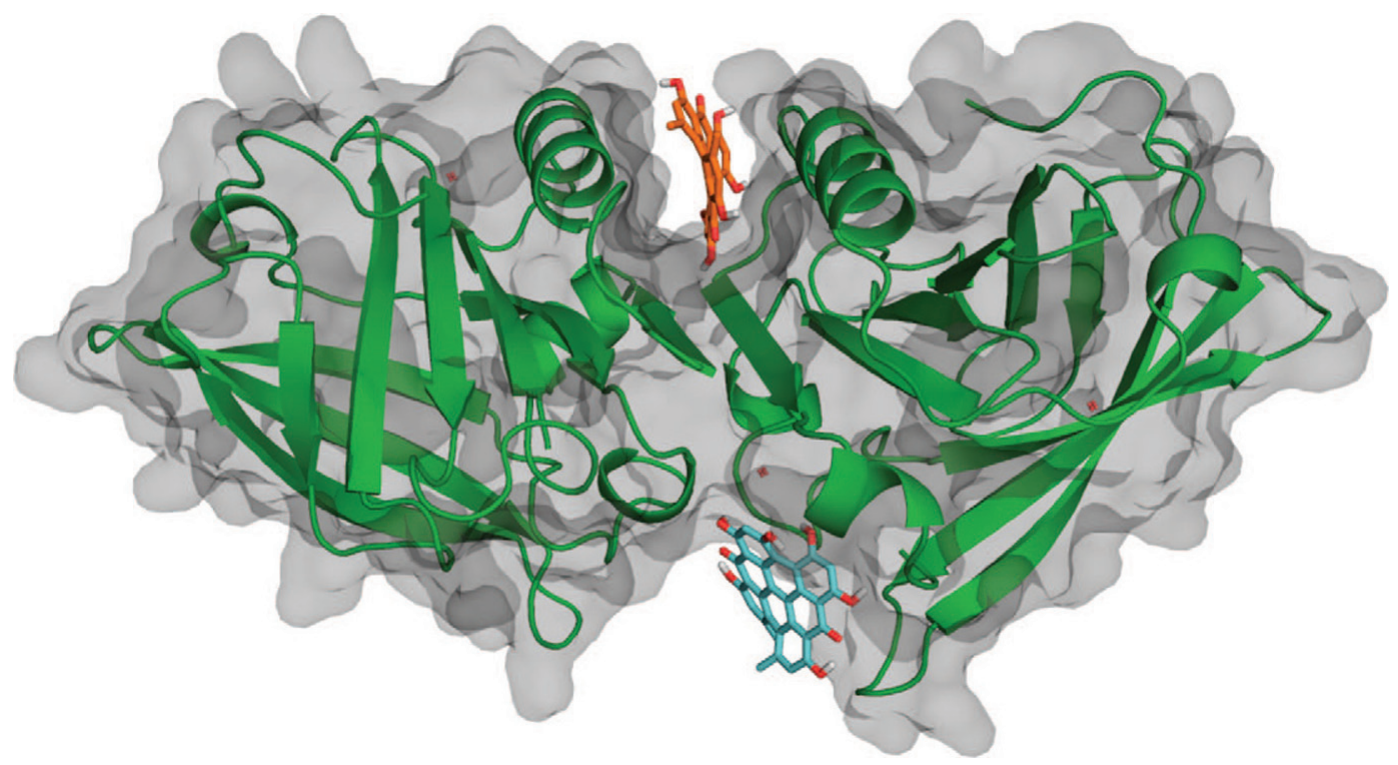

Figure 2. Best poses of hypericin bound to the narrow and wide clefts obtained from docking calculations. Hypericin is shown as sticks [dark gray (orange) and light gray (blue) in the forms bound to the narrow and wide clefts, respectively]. The backbone of the dimeric species of $\beta$-LG is shown in dark gray (green), and the gray area displays the surface of the dimer. Color version available online.

complex in aqueous solutions. Consistent with previous results, it can be clearly observed that the complex has a far superior ability than free Hyp to produce singlet oxygen.

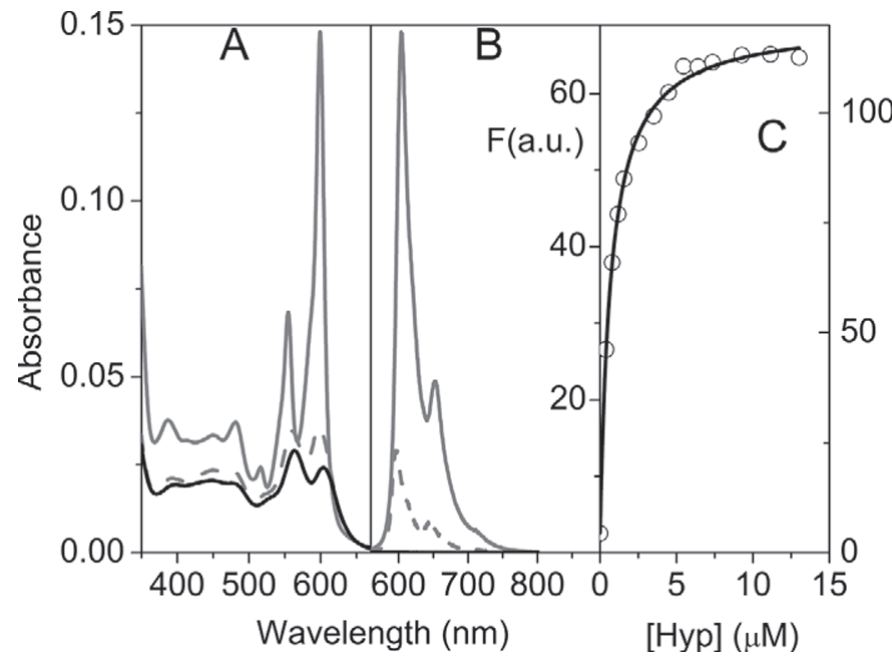

Figure 3. Absorption (A) and fluorescence emission (B) spectra of hypericin solutions $(7 \mu M)$ in dimethylsulfoxide (solid gray), in PBS (solid black), and in PBS in the presence of $100 \mu M \beta$-LG (dashed). (C) Fluorescence emission intensity (estimated from the integrated area) as a function of hypericin concentration at $\beta$-LG $=100 \mu M$ (circles). Assuming a dimerization constant, $K_{D}=8.6 \mu \mathrm{M}^{-1}$, this corresponds to $2 \beta-\mathrm{LG} \approx 40 \mu \mathrm{M}$. The solid line is the result of the fit with a model for a single binding site for hypericin on 23-LG (ComasBarceló et al., 2013) and afford an association constant, $K_{a}=1.40 \pm$ $0.07 \mu M^{-1}$, corresponding to a dissociation constant of $K_{d}=0.71 \pm$ $0.03 \mu M$.

Journal of Dairy Science Vol. 98 No. 1, 2015

\section{Photodynamic Inactivation of Staph. aureus}

Hypericin is widely used in antimicrobial photodynamic therapy due to its bactericidal and fungicidal effect (Rezusta et al., 2012; Yow et al., 2012). To assess the corresponding effects of the Hyp-2 $\beta-\mathrm{LG}$ complex, photodynamic inactivation tests were carried out on Staph. aureus bacteria. The results were compared with those obtained for free Hyp under the same experimental conditions (Figure 5). It can be seen that the complex is devoid of any dark toxicity, yet it is capable

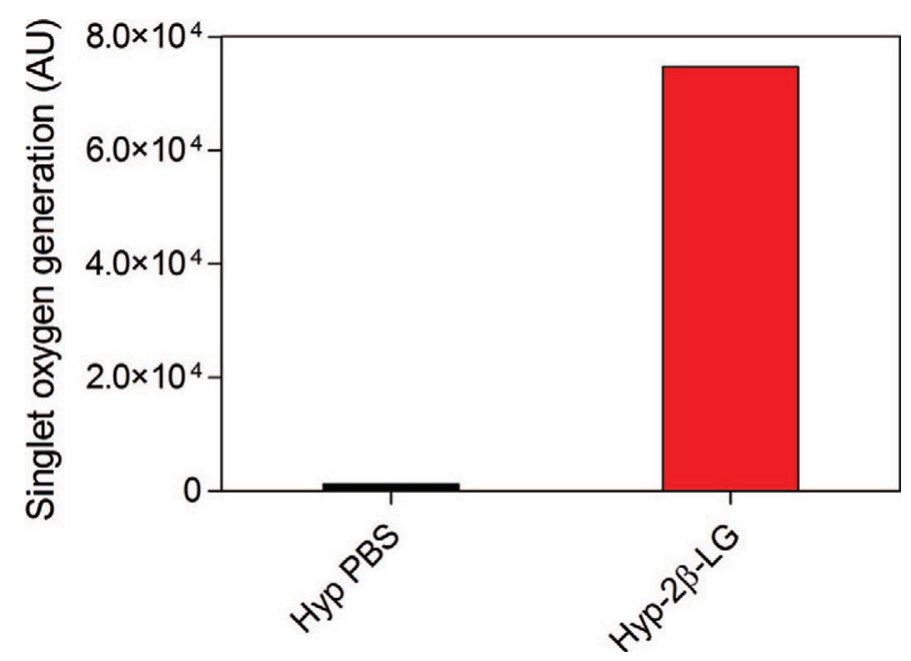

Figure 4. Production of singlet oxygen by hypericin and the complex hypericin-2 $\beta-L G$ in PBS. Concentrations: hypericin $(8 \mu M)$ and $\beta-\mathrm{LG}(40 \mu M)$. Color version available online. 


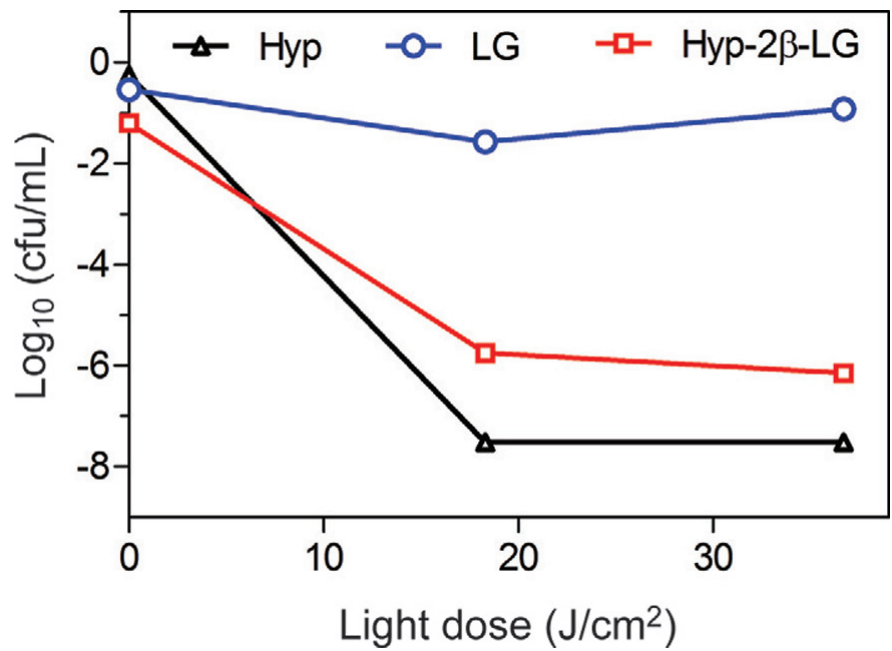

Figure 5. Light dose effects on Staphylococcus aureus photoinactivation by the complex hypericin-2 $\beta$-LG for hypericin $(8 \mu M)$ and $\beta-\mathrm{LG}$ $(40 \mu M)$. Color version available online.

of eradicating bacteria ( 5 logs) upon exposure to visible light. Although the bacterial phototoxicity of Hyp-2 $\beta-$ LG is not much different from that of free Hyp, a major advantage of the use of the Hyp-2 $\beta$-LG complex is that it is administered to the cell suspension using a buffered aqueous solution, which is clearly more compatible with the use in a food processing environment.

\section{CONCLUSIONS}

The complex between Hyp and $\beta$-LG preserves the singlet oxygen photosensitizing properties of Hyp. The nanostructured material is effective against Staph. aureus suspensions and can decrease the bacterial load by 5 orders of magnitude (99.999\%) upon exposure to visible light. The combination of the superb antimicrobial properties of natural hypericin with the full compatibility of the protein carrier with dairy industry processes suggests that it has the potential to be introduced as an effective disinfectant for food manufacturing and handling materials. Further, this APBT might find an application in the routine of milking.

\section{ACKNOWLEDGMENTS}

Financial support for this research was obtained from the Spanish MINECO (CTQ2013-48767-C31-R; SAF2011-27642), Generalitat de Catalunya (2014SGR304 and 2014SGR1189; ICREA Academia), and from Ministero degli Affari Esteri, Direzione generale per la promozione del sistema Paese (Progetti di Grande Rilevanza, Italia-Argentina 2011-2013). J.Juárez-Jiménez thanks Instituto de Salud Carlos III for FIS fellowship. The Consorci de Serveis Universitaris de Catalunya is acknowledged for computational facilities.

\section{REFERENCES}

Åkerström, B., N. Borregaard, D. R. Flower, and J. P. Salier. 2006. Lipocalins. Landes Bioscience, Austin, TX.

Brockmann, H., M. N. Haschad, K. Maier, and F. Pohl. 1939. Hypericin, the photodynamically active pigment from Hypericum perforatum. Naturwissenschaften 32:550.

Brownlow, S., J. H. M. Cabral, R. Cooper, D. R. Flower, S. J. Yewdall, I. Polikarpov, A. C. T. North, and L. Sawyer. 1997. Bovine betalactoglobulin at 1.8 angstrom resolution-Still an enigmatic lipocalin. Structure 5:481-495.

Case, D. A., T. A. Darden, I. T. E. Cheatham, C. L. Simmerling, J. Wang, R. E. Duke, R. Luo, R. C. Walker, W. Zhang, K. M. Merz, B. Roberts, S. Hayik, A. Roitberg, G. Seabra, J. Swails, A. W. Götz, I. Kolossváry, K. F. Wong, F. P. J. Vanicek, R. M. Wolf, J. Liu, X. Wu, S. R. B. T. Steinbrecher, H. Gohlke, Q. Cai, X. Ye, J. Wang, M.-J. Hsieh, G. Cui, D. R. Roe, D. H. Mathews, M. G. Seetin, R. Salomon-Ferrer, C. Sagui, V. Babin, T. Luchko, S. Gusarov, and A. K. P. A. Kollman. 2012. AMBER12. University of California, San Francisco.

Comas-Barceló, J., B. Rodríguez-Amigo, S. Abbruzzetti, P. d. Rey-Puech, M. Agut, S. Nonell, and C. Viappiani. 2013. A self-assembled nanostructured material with photosensitising properties. RSC Adv. 3:17874-17879.

Das, K., A. V. Smirnov, J. Wen, P. Miskovsky, and J. W. Petrich. 1999. Photophysics of hypericin and hypocrellin a in complex with subcellular components: Interactions with human serum albumin. Photochem. Photobiol. 69:633-645.

Demirci, A., and M. O. Ngadi. 2012. Microbial decontamination in the food industry: Novel methods and applications. Pages 1-804 in Series in Food Science, Technology and Nutrition. Vol. 234 Woodhead Publishing, Philadelphia, PA.

Doyle, M. E., F. A. Hartmann, and A. C. L. Wong. 2011. White Paper on Sources of Methicillin-Resistant Staphylococcus aureus (MRSA) and Other Methicillin-Resistant Staphylococci: Implications for Our Food Supply? FRI Food Safety Reviews, University of Wisconsin, Madison.

Durán, N., and P. S. Song. 1986. Hypericin and its photodynamic action. Photochem. Photobiol. 43:677-680.

Fehr, M. J., M. A. McCloskey, and J. W. Petrich. 1995. Light-induced acidification by the antiviral agent hypericin. J. Am. Chem. Soc. 117:1833-1836.

Flower, D. R., A. C. T. North, and C. E. Sansom. 2000. The lipocalin protein family: structural and sequence overview. Biochim. Biophys. Acta-Protein Struct. Mol. Enzym. 1482:9-24.

Friesner, R. A., J. L. Banks, R. B. Murphy, T. A. Halgren, J. J. Klicic, D. T. Mainz, M. P. Repasky, E. H. Knoll, M. Shelley, J. K. Perry, D. E. Shaw, P. Francis, and P. S. Shenkin. 2004. Glide: A new approach for rapid, accurate docking and scoring. 1. Method and assessment of docking accuracy. J. Med. Chem. 47:1739-1749.

Graveland, H., B. Duim, E. vanDuijkeren, D. Heederik, and J. A. Wagenaar. 2011. Livestock-associated methicillin-resistant Staphylococcus aureus in animals and humans. Int. J. Med. Microbiol. 301:630-634.

Halgren, T. A., R. B. Murphy, R. A. Friesner, H. S. Beard, L. L. Frye, W. T. Pollard, and J. L. Banks. 2004. Glide: A new approach for rapid, accurate docking and scoring. 2. Enrichment factors in database screening. J. Med. Chem. 47:1750-1759.

Hornak, V., R. Abel, A. Okur, B. Strockbine, A. Roitberg, and C. Simmerling. 2006. Comparison of multiple Amber force fields and development of improved protein backbone parameters. Proteins 65:712-725.

IDF. 2013. Guide to Prudent Use of Antimicrobial Agents in Dairy Production. Federation Internationale du Lait-International Dairy Federation (IDF), Brussels, Belgium. 
Jacobson, J. M., L. Feinman, L. Liebes, N. Ostrow, V. Koslowski, A. Tobia, B. E. Cabana, D. H. Lee, J. Spritzler, and A. M. Prince. 2001. Pharmacokinetics, Safety, and antiviral effects of hypericin, a derivative of St. John's wort plant, in patients with chronic hepatitis $\mathrm{C}$ virus infection. Antimicrob. Agents Chemother. 45:517-524.

Jiménez-Banzo, A., X. Ragàs, P. Kapusta, and S. Nonell. 2008. Timeresolved methods in biophysics. 7. Photon counting vs. analog time-resolved singlet oxygen phosphorescence detection. Photochem. Photobiol. Sci. 7:1003-1010.

Jori, G., M. Camerin, M. Soncin, L. Guidolin, and O. Coppellotti. 2011. Chapter 1 Antimicrobial Photodynamic Therapy: Basic Principles. Pages 1-18 in Photodynamic Inactivation of Microbial Pathogens: Medical and Environmental Applications. Vol. 11. The Royal Society of Chemistry, Cambridge, UK.

Kairyte, K., S. Lapinskas, V. Gudelis, and Z. Luksiene. 2012. Effective inactivation of food pathogens Listeria monocytogenes and Salmonella enterica by combined treatment of hypericin-based photosensitization and high power pulsed light. J. Appl. Microbiol. 112:1144-1151.

Kubin, A., F. Wierrani, U. Burner, G. Alth, and W. Grünberger. 2005. Hypericin-The facts about a controversial agent. Curr. Pharm. Des. 11:233-253.

López-Chicón, P., M. P. Paz-Cristobal, A. Rezusta, C. Aspiroz, M. Royo-Cañas, E. Andres-Ciriano, Y. Gilaberte, M. Agut, and S. Nonell. 2012. On the mechanism of Candida spp. photoinactivation by hypericin. Photochem. Photobiol. Sci. 11:1099-1107.

Luksiene, Z., and L. Brovko. 2013. Antibacterial photosensitizationbased treatment for food safety. Food Eng. Rev. 5:185-199.

Mercadante, D., L. D. Melton, G. E. Norris, T. S. Loo, M. A. K. Williams, R. C. J. Dobson, and G. B. Jameson. 2012. Bovine $\beta$-lactoglobulin is dimeric under imitative physiological conditions: dissociation equilibrium and rate constants over the $\mathrm{pH}$ range of 2.5-7.5. Biophys. J. 103:303-312.
Michaeli, A., A. Regev, Y. Mazur, J. Feitelson, and H. Levanon. 1993. Triplet state reactions of hypericin. Time-resolved laser photolysis and electron paramagnetic resonance spectroscopy. J. Phys. Chem. 97:9154-9160.

Miskovsky, P., D. Jancura, S. Sanchez-Cortez, E. Kocisova, and L. Chinsky. 1998. Antiretrovirally active drug hypericin binds the iia subdomain of human serum albumin: A resonance raman and surface-enhanced raman spectroscopy study. J. Am. Chem. Soc. 120:6374-6379.

Nafee, N., A. Youssef, H. El-Gowelli, H. Asem, and S. Kandil. 2013. Antibiotic-free nanotherapeutics: Hypericin nanoparticles thereof for improved in vitro and in vivo antimicrobial photodynamic therapy and wound healing. Int. J. Pharm. 454:249-258.

Ogilby, P. R. 2010. Singlet oxygen: There is indeed something new under the sun. Chem. Soc. Rev. 39:3181-3209.

Rezusta, A., P. López-Chicón, M. P. Paz-Cristobal, M. Alemany-Ribes, D. Royo-Díez, M. Agut, C. Semino, S. Nonell, M. J. Revillo, C. Aspiroz, and Y. Gilaberte. 2012. In vitro fungicidal photodynamic effect of hypericin on Candida species. Photochem. Photobiol. 88:613-619.

Tanford, C., and Y. Nozaki. 1959. Physico-chemical comparison of $\beta$-lactoglobulins A and B. J. Biol. Chem. 234:2874-2877.

Trevisi, E., A. Zecconi, S. Cogrossi, E. Razzuoli, P. Grossi, and M. Amadori. 2014. Strategies for reduced antibiotic usage in dairy cattle farms. Res. Vet. Sci. 96:229-233.

Yamazaki, T., N. Ohta, I. Yamazaki, and P. S. Song. 1993. Excitedstate properties of hypericin: electronic spectra and fluorescence decay kinetics. J. Phys. Chem. 97:7870-7875.

Yow, C. M. N., H. M. Tang, E. S. M. Chu, and Z. Huang. 2012. Hypericin-mediated photodynamic antimicrobial effect on clinically isolated pathogens. Photochem. Photobiol. 88:626-632. 